\title{
MicroRNA-207 enhances radiation-induced apoptosis by directly targeting akt3 in cochlea hair cells
}

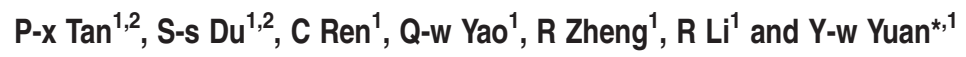

MicroRNAs (miRNAs) have important roles in various types of cellular biological processes. Our study aimed to determine whether miRNAs function in the regulation of ionizing radiation (IR)-induced cell death in auditory cells and to determine how they affect the cellular response to IR. Microarray and qRT-PCR were performed to identify and confirm the differential expression of miRNAs in the cochlea hair cell line HEI-OC1 and in vivo after IR. Upregulation or downregulation of miRNAs using miRNA mimics or inhibitor were detected to characterize the biological effects of the indicated miRNAs. Bioinformatic analyses, luciferase reporter assays and mRNA knockdown were performed to identify a miRNA target gene. We determined that miR-207 was significantly upregulated after IR. MiR-207 enhances IR-induced apoptosis and DNA damage in HEI-OC1 cells. Furthermore, Akt3 was confirmed to be a direct target of miR-207. Downregulation of Akt3 mimics the effects of miR-207. MiR-207 enhances IR-induced apoptosis by directly targeting Akt3 and anti-miR-207 may have a potential role in protecting cochlea hair cells from IR.

Cell Death and Disease (2014) 5, e1433; doi:10.1038/cddis.2014.407; published online 2 October 2014

Radiotherapy (RT) is one of the most important treatments for head and neck (HN) cancers. Although the technologies for RT have greatly improved in recent years, the incidence of side effects induced by RT remains high. Sensorineural hearing loss (SNHL) is considered to be a principal complication of RT for $\mathrm{HN}$ and markedly affects the quality of life for patients with HN cancers. ${ }^{1}$ It has been demonstrated that the death of cochlea hair cells is responsible for ionizing radiation (IR)induced SNHL. ${ }^{2-6}$ Regulators, such as p53, reactive oxygen species and c-Jun $\mathrm{N}$-terminal kinases are known to have important roles in apoptosis of irradiated hair cells. ${ }^{7-10}$ $\mathrm{N}$-acetylcysteine, ${ }^{11}$ epicatechin ${ }^{10}$ and metformin ${ }^{12}$ have been previously demonstrated to be effective in reducing apoptosis in irradiated hair cells. Because studies have been limited, the mechanisms underlying IR-induced auditory cell death remain unclear and require further investigation.

MicroRNAs (miRNAs) are a class of short noncoding RNAs. Some miRNAs have been extensively investigated. These RNAs repress translation or the stability of target mRNAs by binding to the 3'-untranslated regions (UTRs) of the mRNAs with imperfect complementarity. ${ }^{13,14}$ By regulating gene expression at the post-transcriptional level, miRNAs have important roles in various types of cellular biological processes, including responses to IR. However, most published studies on miRNAs regulating radiosensitivity have examined cancer cells, and little attention has been devoted to normal cells. Moreover, the relationship between miRNAs and IR-induced cochlea hair cell death has not been investigated to date.

In this study, we aimed to investigate IR-responsive miRNAs by analyzing the miRNA expression profile in the auditory cell line $\mathrm{HEI}-\mathrm{OC} 1$ and identified miR-207 as an IR-inducible
miRNA. MiR-207 enhanced apoptosis by increasing DNA damage in irradiated $\mathrm{HEI}-\mathrm{OC} 1$ cells. Further investigation revealed that miR-207 negatively regulated Akt3 as a direct target. Thus, we provide a new mechanism for IR-induced apoptosis in cochlea hair cells. Taken together, our findings may help to develop a potential protectant for IRinduced SNHL.

\section{Results}

MiR-207 expression is induced by IR and inhibits cell growth. A differential miRNA expression profile between irradiated and nonirradiated $\mathrm{HEI}-\mathrm{OC} 1$ cells was determined using microarray. These results revealed that three miRNAs were upregulated, and nine miRNAs were downregulated after IR when taking into account a fold-change $>6.3$ (Ig0.8) and $P<0.05$ (Table 1). We focused on the three upregulated miRNAs, including miR-207, miR-29c and miR-466i-5p, for further investigation. The qRT-PCR results confirmed that all these miRNAs were significantly upregulated at 12, 24 and $48 \mathrm{~h}$ after IR and the high expression remained stable (Figure 1a). After we successfully transfected miR-207, miR-29c and miR-466i-5p mimics individually into HEI-OC1 cells (Figure 1b), an methyl thiazolyl tetrazolium (MTT) assay was performed. Upregulation of miR-207 significantly inhibited cell growth in cells after IR (10,20 Gy) but not in cells without IR, whereas miR-29c or miR-466i-5p overexpression did not exhibit any growth effect in irradiated or nonirradiated cells (Figure 1c). To confirm the expression of miR-207 in vivo, we performed assays on irradiated and control cochleas. Results from qRT-PCR, northern blotting and in situ hybridization (ISH) were identical and further verified the

\footnotetext{
${ }^{1}$ Department of Radiation Oncology, Nanfang Hospital, Southern Medical University, Guangzhou, Guangdong 510515, China

*Corresponding author: Y Yuan, Department of Radiation Oncology, Nanfang Hospital, Southern Medical University, No. 1838 Guangzhou DaDao Bei, Guangzhou, Guangdong 510515, China. Tel: +86 206164 2136; Fax: +86 206164 2136; E-mail: yuanyw66@aliyun.com

${ }^{2}$ These authors contributed equally to this work.

Abbreviations: IR, ionizing radiation; RT, radiotherapy; HN, head and neck; SNHL, sensorineural hearing loss; UTRs, untranslated regions; MTT, methyl thiazolyl tetrazolium; ISH, in situ hybridization

Received 02.5.14; revised 06.8.14; accepted 20.8.14; Edited by D Aberdam
} 
upregulation of miR-207 in irradiated cochleas (Figures 1d-f). On the basis of this finding, further studies were performed to determine how miR-207 affects cell growth.

MiR-207 enhances IR-induced apoptosis. The flow cytometry results for cell cycle analysis showed that populations of $\mathrm{G} 1, \mathrm{~S}$ and $\mathrm{G} 2$ phases were not significantly different

Table 1 Differential miRNAs expression in HEI-OC1 cells after irradiation

\begin{tabular}{llrc}
\hline & miRNA ID & Ig (ratio) & \multicolumn{1}{c}{$\boldsymbol{P}$-value } \\
\hline Upregulated & mmu-miR-207 & 1.13 & $3.73 \mathrm{E}-05$ \\
& mmu-miR-29c & 0.96 & $4.96 \mathrm{E}-03$ \\
Downregulated & mmu-miR-466i-5p & 3.00 & $4.51 \mathrm{E}-05$ \\
& mmu-miR-101a-5p & -1.04 & $1.03 \mathrm{E}-05$ \\
& mmu-miR-1247-5p & -1.19 & $2.12 \mathrm{E}-05$ \\
& mmu-miR-1899 & -1.12 & $4.99 \mathrm{E}-06$ \\
& mmu-miR-222 & -1.22 & $1.80 \mathrm{E}-04$ \\
& mmu-miR-3473d & -1.37 & $6.99 \mathrm{E}-05$ \\
& mmu-miR-375-5p & -1.24 & $8.82 \mathrm{E}-06$ \\
& mmu-miR-491-5p & -1.22 & $1.74 \mathrm{E}-04$ \\
& mmu-miR-5100 & -1.09 & $7.67 \mathrm{E}-04$ \\
& mmu-miR-719 & -1.11 & $2.41 \mathrm{E}-04$ \\
\hline
\end{tabular}

between miR-207 transfected and control cells after IR (Figure 2a), which indicated that miR-207 did not affect the distribution of cell cycle in irradiated cells. Next, we investigated whether miR-207 affected apoptosis in HElOC1 cells. The flow cytometry results for apoptosis indicated an upregulation of miR-207 significantly enhanced apoptosis compared with control in irradiated cells, whereas inhibition of miR-207 significantly mitigated apoptosis (Figure 2b). In cells without IR, no differences were found between groups treated with miR-207, miR-207 inhibitor or control. To confirm the apoptosis-enhancement effect of miR-207, western blotting analyses were performed. MiR-207 moderately increased the expression of cleaved PARP after IR, whereas inhibition of miR-207 greatly repressed cleaved PARP expression (Figure 2c). Furthermore, in cells treated without IR, the level of miR-207 did not affect the expression of cleaved PARP. On the basis of these studies, we concluded that miR-207 enhanced apoptosis, which only occurred in cells with IR.

MiR-207 enhances IR-induced DNA damage. Next, we investigated whether increased apoptosis by miR-207 is
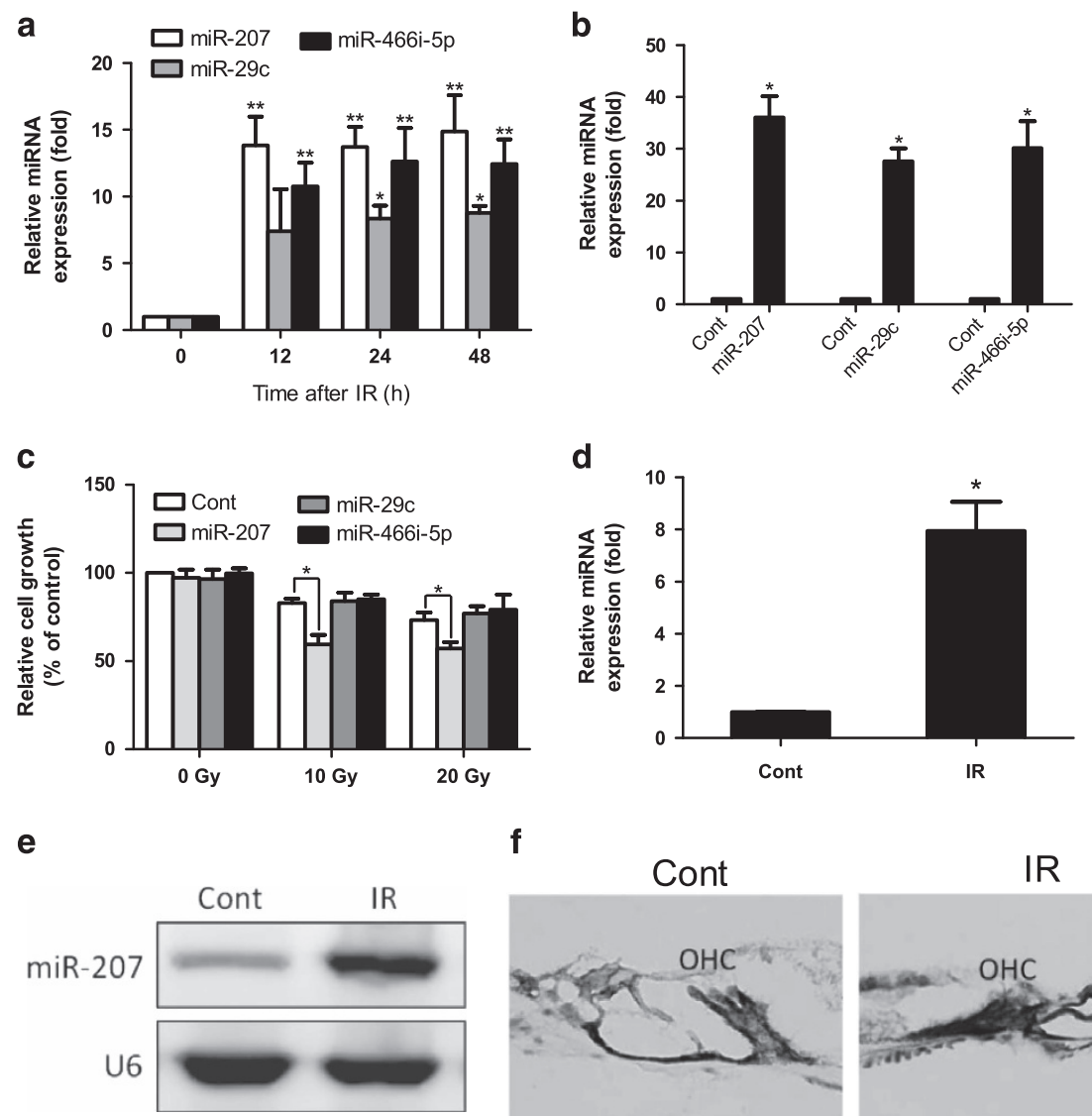

f
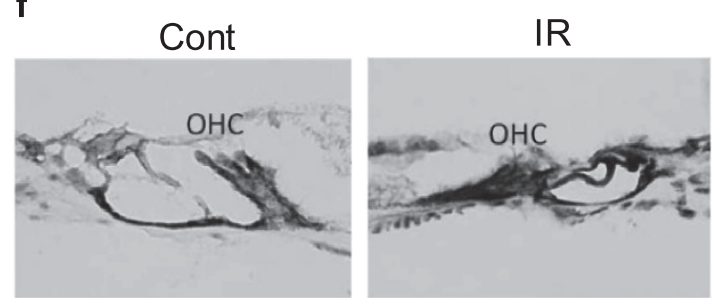

Figure 1 miR-207 expression is induced by IR and inhibits cell growth. (a) qRT-PCR was performed to confirm the upregulated expression of miR-207, miR-29c and miR-466i-5p in HEI-OC1 cells at 12, 24 and $48 \mathrm{~h}$ after 20 Gy irradiation. U6 spliceosomal RNA was used for normalization. Error bar, S.D.; ${ }^{*} P<0.05$ ( $n=3$ ) versus the nonirradiated $(0 \mathrm{~h})$ group, ${ }^{* \star} P<0.001(n=3)$ versus the nonirradiated $(0 \mathrm{~h})$ group. (b) HEl-OC1 cells were transfected with miR-207, miR-29c and miR-466i-5p independently. qRT-PCR was performed to detect the level of the indicated miRNAs at $48 \mathrm{~h}$ after transfection. Error bar, S.D.; ${ }^{*} P<0.01(n=3)$ versus control. (c) HEl-OC1 cells transfected with miR-207, miR-29c, miR-466i-5p and control miRNAs were subjected to the MTT assay at $24 \mathrm{~h}$ after IR (10, 20 Gy) or without IR. Error bar, S.D.; ${ }^{*} P<0.001$ ( $n=3$ ) versus control. (d) qRT-PCR, (e) northern blotting and (f) in situ hybridization were performed to confirm the upregulated expression of miR-207 in cochlea at $24 \mathrm{~h}$ after $20 \mathrm{~Gy}$ irradiation on mice. U6 was used for normalization. Error bar, S.D.; ${ }^{*} P<0.001(n=5)$ versus control. $\mathrm{OHC}=$ outer hair cell 
a

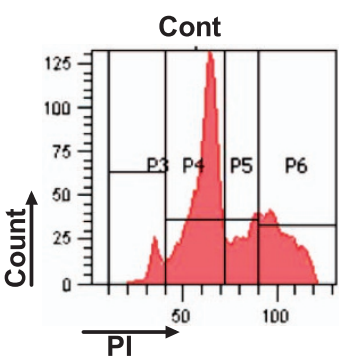

miR-207

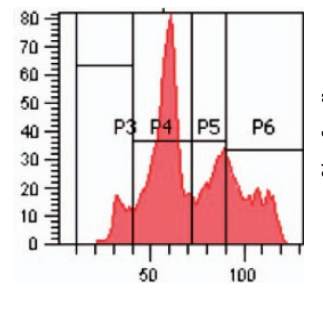

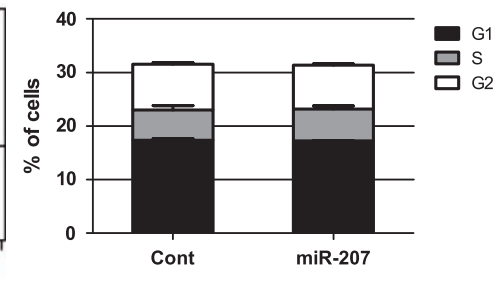

b
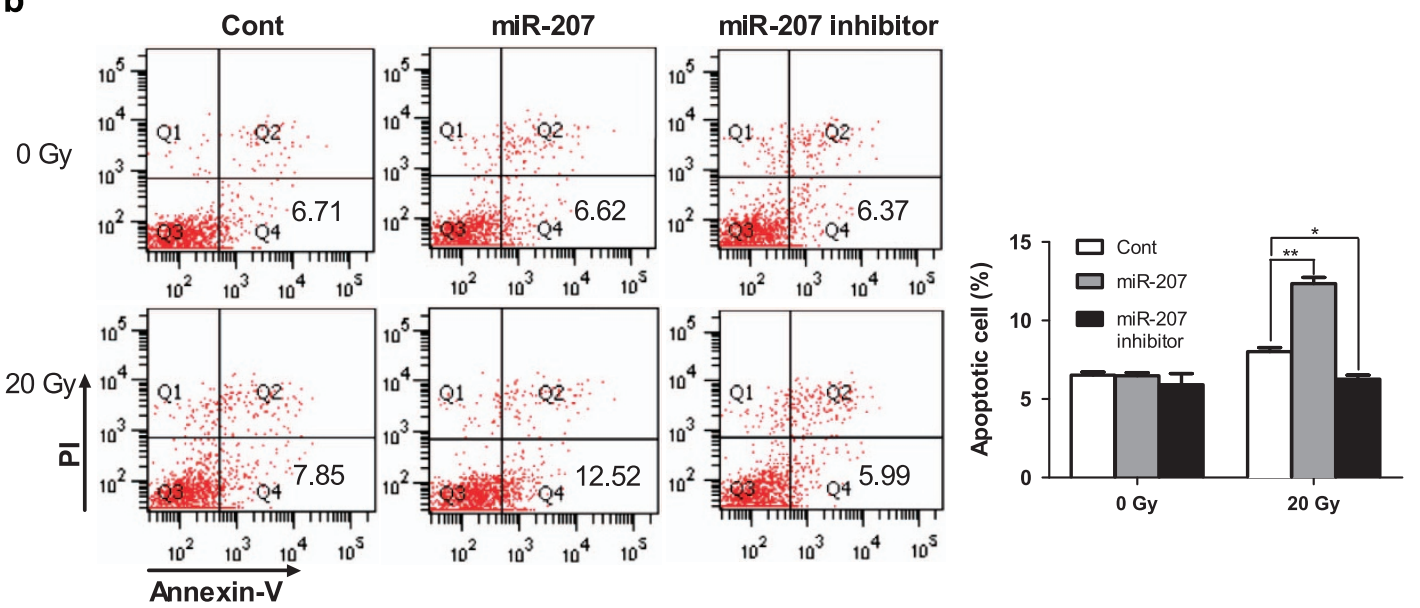

C

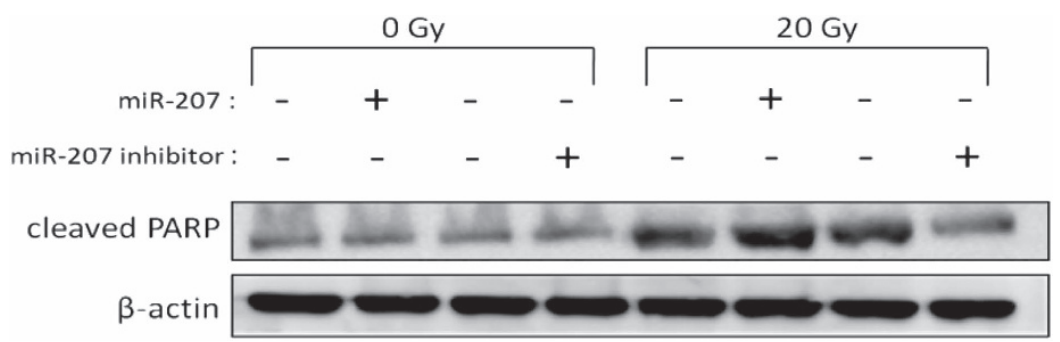

Figure 2 miR-207 enhances IR-induced apoptosis. HEl-OC1 cells were transfected with miR-207, miR-207 inhibitor or control miRNAs prior to subsequent experiments. (a) Cell cycle analysis was performed $24 \mathrm{~h}$ after IR (20 Gy) to examine the effect of miR-207 on cell cycle distribution. (b) The apoptosis assay using flow cytometry was performed $24 \mathrm{~h}$ after transfected cells were treated with 0 Gy IR or without IR. Error bars, S.D.; ${ }^{*} P=0.001(n=3)$ versus the control group, ${ }^{* *} P<0.001(n=3)$ versus control. (c) The expression levels of cleaved PARP were detected by western blotting analyses using an anti-cleaved PARP antibody in transfected cells at $24 \mathrm{~h}$ after $20 \mathrm{~Gy}$ IR or without IR

associated with an enhancement in DNA damage. Transfection with miR-207 resulted in higher $\gamma$-H2AX expression at 6 and $12 \mathrm{~h}$ after IR compared with control. In contrast, transfection with miR-207 inhibitors resulted in significantly lower $\gamma$-H2AX expression independent of time (6 or $12 \mathrm{~h}$ ) after IR (Figure 3a). We also assessed the DNA damage by quantifying $\gamma$-H2AX foci after staining. HEI-OC1 cells with upregulated miR-207 expression showed significantly more foci in the nucleus, whereas cells with downregulated miR-207 expression showed the opposite result. Taken together, these results suggest that miR-207 significantly enhanced IR-induced DNA damage.

Akt3 is a direct target of miR-207. To identify the target mRNA of miR-207, we used five miRNA target prediction programs, ${ }^{15}$ including EIMMo, miRanda, miTarget, PicTar and TargetScan. As shown in Figure $4 \mathrm{a}$, the complementary sequence for the seed region of miR-207 was at position
1184-1191 of Akt3 3'-UTR. A reporter (WT 3'-UTR) containing the exact complementary sequence in the Akt3 3 '-UTR fragment and another reporter (Mut 3'-UTR) containing mutated nucleotides of the complementary sequence were constructed (Figure 4a). Expectedly, co-transfection with miR-207 and WT 3'-UTR reporter significantly reduced luciferase activity compared with control (Figure 4b). In addition, Akt3 mRNA and protein levels significantly decreased after miR-207 transfection (Figures 4c and d). However, the expression of other Akt isoforms, including Akt1 and Akt2, remained unchanged (Figure 4d). Moreover, P-Akt increased markedly after IR, but decreased moderately in cells transfected with miR-207. Taken together, these results revealed that Akt3 is a direct target of miR-207.

Downregulation of Akt3 mimics the effects of miR-207. We next examined whether downregulation of Akt3 exhibited similar effects of miR-207. First, we specifically 
a

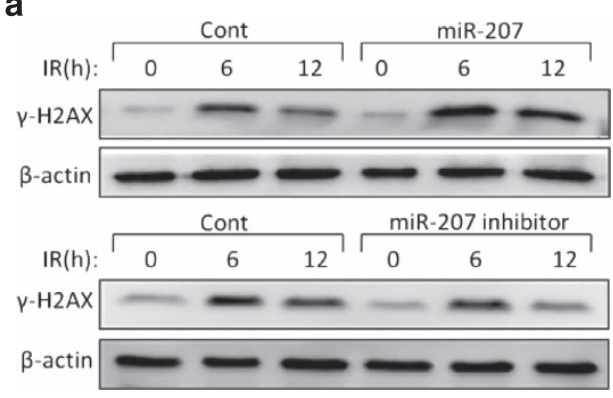

b
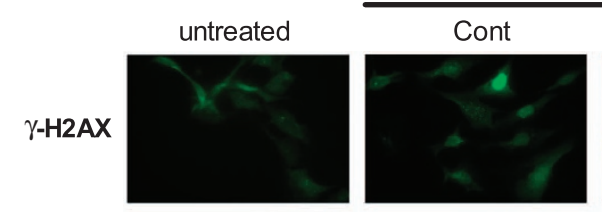

20 Gy IR
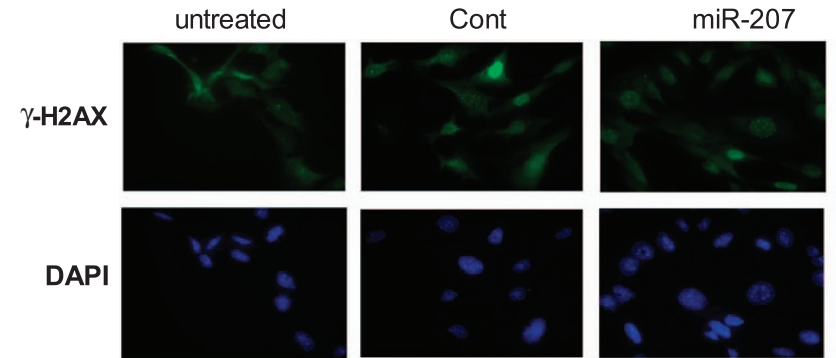

miR-207 inhibitor
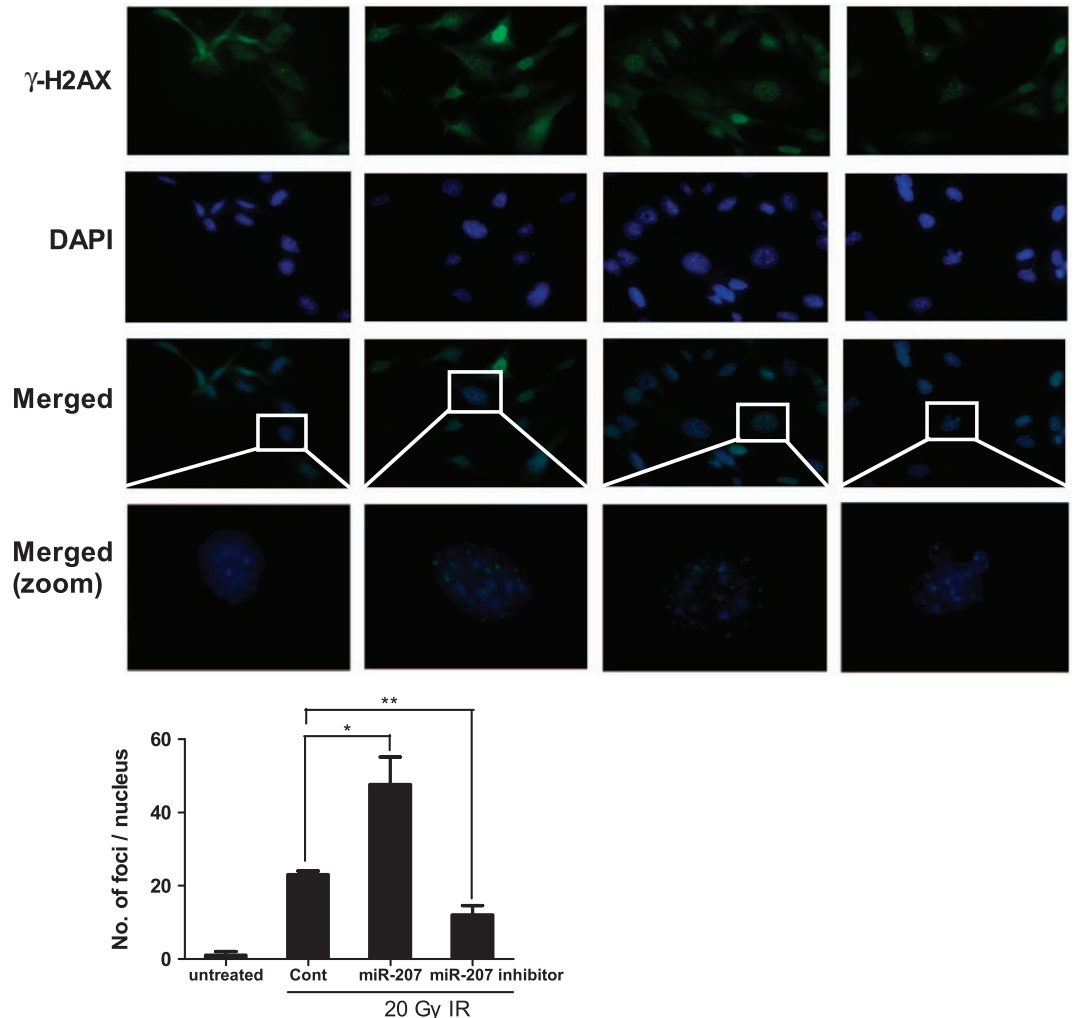

Figure 3 miR-207 enhances IR-induced DNA damage. HEI-OC1 cells were transfected with miR-207, miR-207 inhibitor or control miRNAs prior to subsequent experiments (a) Transfected cells were treated with $20 \mathrm{~Gy}$ IR or without IR, and collected at 6 and $12 \mathrm{~h}$ after IR. Expression levels of $\gamma$-H2AX (green) were detected by western blotting analyses using an anti- $\gamma-\mathrm{H} 2 \mathrm{AX}$ antibody. (b) Immunofluorescent staining for $\gamma-\mathrm{H} 2 \mathrm{AX}$ was performed at $12 \mathrm{~h}$ after $20 \mathrm{~Gy}$ IR of transfected cells. Error bar, S.D.; ${ }^{*} P=0.001(n=3)$ versus control; ${ }^{* \star} P<0.05(n=3)$ versus control

repressed the expression of Akt3 protein, but not Akt1 or Akt2 (Figure 5a). Apoptosis analysis showed that HEI-OC1 cells transfected with miR-207 or siAkt3 exhibited significantly greater apoptosis compared with control (Figure 5b). At the protein level, western blotting analyses detected the expression of $\gamma-\mathrm{H} 2 \mathrm{AX}$ as well as cleaved PARP, which increased similarly between cells transfected with miR-207 and siAkt3 (Figure 5c).

\section{Discussion}

MiRNAs are indispensible for cochlea hair cell maintenance and survival. ${ }^{16}$ The expression of miRNA changes while the cochlea is under various stress, eventually causing cell death and SNHL. ${ }^{17}$ Oxidative stress upregulates 35 miRNAs and downregulates 40 miRNAs in HEl-OC1 cells. ${ }^{18}$ The miR-183/ Taok1 target pair and miR-34 family are found implicated in cochlear responses to acoustic trauma and kanamycin ototoxicity, respectively. ${ }^{19,20}$ In our study, miR-207, miR-29c and miR-466i-5p were identified as upregulated miRNAs in $\mathrm{HEI}-\mathrm{OC} 1$ cells after IR, and miR-207 was confirmed to be the only one that affects cell viability. These evidences show that different stress may cause different miRNA expression in cochlea cells, which is probably because different miRNAs take part in different cellular processes. To the best of our knowledge, miR-207 has not been thoroughly investigated. MiR-207 was found to be downregulated in liver tissue after partial hepatectomy in mice ${ }^{21}$ and upregulated in a neuronal 


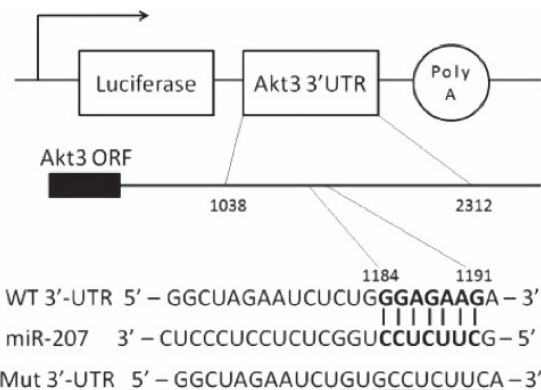

C

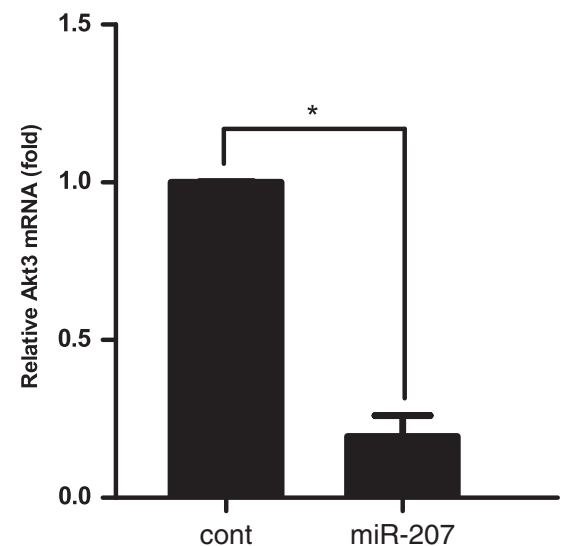

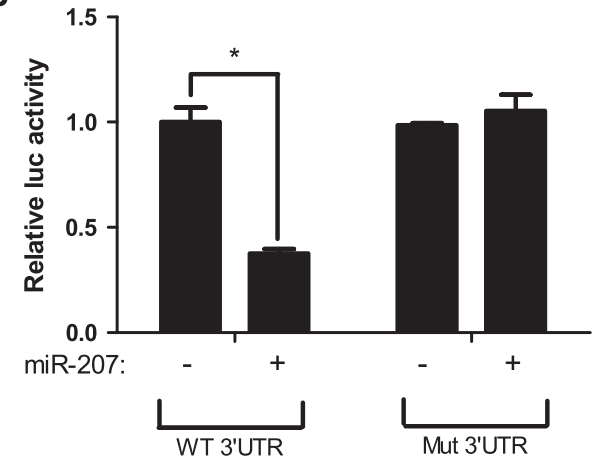

d miR-207:

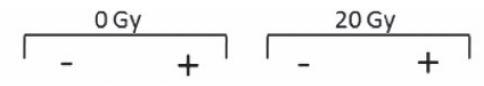

Akt3

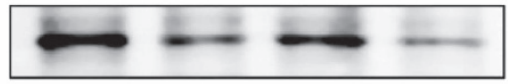

Akt2

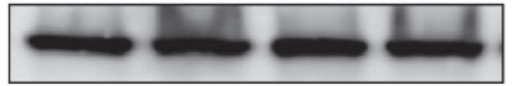

Akt1

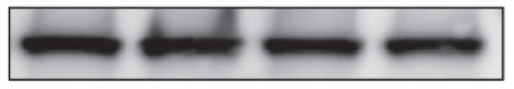

P-Akt

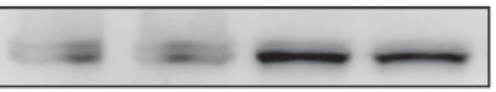

$\beta$-actin

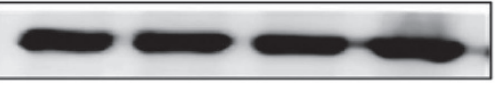

Figure 4 Akt3 is a direct target of miR-207. (a) The structure of the reporter plasmid, nucleotide of Akt3 3'-UTR complementary to the seed sequence of miR-207 and the mutant sequence. (b) The relative luciferase activity in HEI-OC1 cells was determined after co-transfection with Akt3 3'-UTR or Mut-Akt3 3'-UTR plasmids and miR-207 or control. For each sample, the relative luciferase activity was normalized with firefly luciferase activity. Error bars, S.D.; ${ }^{*} P=0.001$ ( $n=3$ ) versus control. (c) Akt3 mRNA expression was determined using qRT-PCR in cells transfected with miR-207 or control miRNAs. Error bars, S.D.; ${ }^{*} P<0.01$ ( $n=3$ ) versus control. (d) Expression levels of Akt3 and other related proteins were determined by western blotting analyses using indicated antibodies in miR-207 or control miRNA-transfected cells. The transfected cells were collected at $24 \mathrm{~h}$ after 20 Gy IR or without IR

cell line (MN9D) with 6-hydroxydopamine (6-OHDA) treatment, a component of a neurotoxin. ${ }^{22}$ Although these studies demonstrated changes in miR-207 expression, they did not investigate the function of miR-207. Our study reveals the biological function of miR-207 and proposes a miRNA correlated to IR-induced injury in auditory cells.

Further studies have revealed that inhibition of cell growth by miR-207 is caused by increased cell apoptosis rather than cell cycle arrest. Moreover, an enhancement of apoptosis by miR-207 was only observed in irradiated cells, which suggested that this change is associated with IR-induced DSBs. It is known that DNA is the major target of radiation effects. The unsuccessful repair of DSBs may result in lethal consequences, such as apoptosis, for cells. ${ }^{23}$ Moreover, $\gamma-\mathrm{H} 2 \mathrm{AX}$ is one of the earliest markers of DSBs after IR. ${ }^{24}$ In the present study, the expression of $\gamma-\mathrm{H} 2 \mathrm{AX}$ was greatly increased and was sustained in cells upregulated with miR-207. This finding indicated that miR-207 enhanced IRinduced DNA damage, which results in enhanced apoptosis.

Akt3 has been shown to be a direct target of miR-207 in our study. Isoforms of the Akt family, including Akt3, Akt1 and Akt2, share a high degree of structural similarity, but express and function differently in specific cell types and biological processes. Akt3 has been studied extensively in cancer cells for its effects on cancer development, proliferation and migration; ${ }^{25,26}$ however, little is known regarding its function in normal cells. In our study, Akt3 was found to be specifically inhibited by miR-207. Inhibition by siAkt3 resulted in an enhancement of apoptosis and DSBs in irradiated HEI-OC1 cells, similar to results obtained with miR-207 treatment. This indicated Akt3 potentially has an important role in miR-207mediated IR response in auditory cells. In addition, Akt1 and Akt2 were shown to be important regulators in the activation of DNA-dependent protein kinase, a key enzyme of nonhomologous end joining of the DNA repair pathway. ${ }^{27-29}$ Akt3 may share a DSB repair pathway with Akt1 and Akt2, and further studies are required.

As previously mentioned, the therapeutic options against IR-induced cochlea hair cell death are notably limited. Thus, the identification of new therapeutic agents is important. Although we have identified many genes related to apoptosis induced by IR, it is not easy to directly manipulate these genes. Among the regulators of protein-coding genes, miRNA is an ideal choice. In our study, specific inhibition of miR-207 exhibits the exciting potential to protect $\mathrm{HEI}-\mathrm{OC} 1$ cells from IR by reducing apoptosis and DSBs. Thus, further studies are required to confirm the protective effect of anti-miR-207 in vivo. 
a

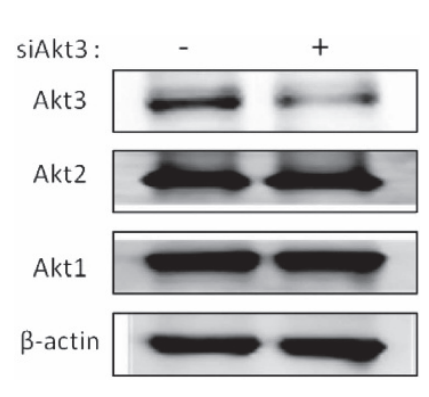

C

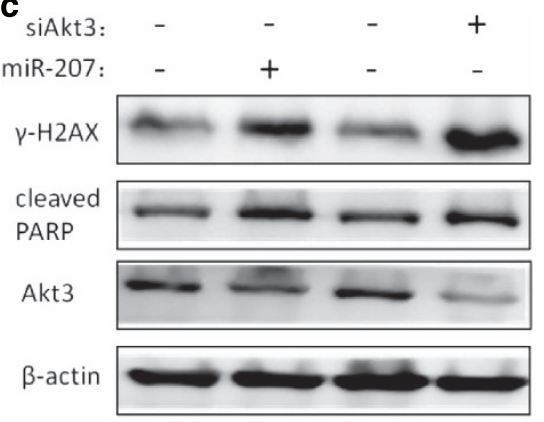

b
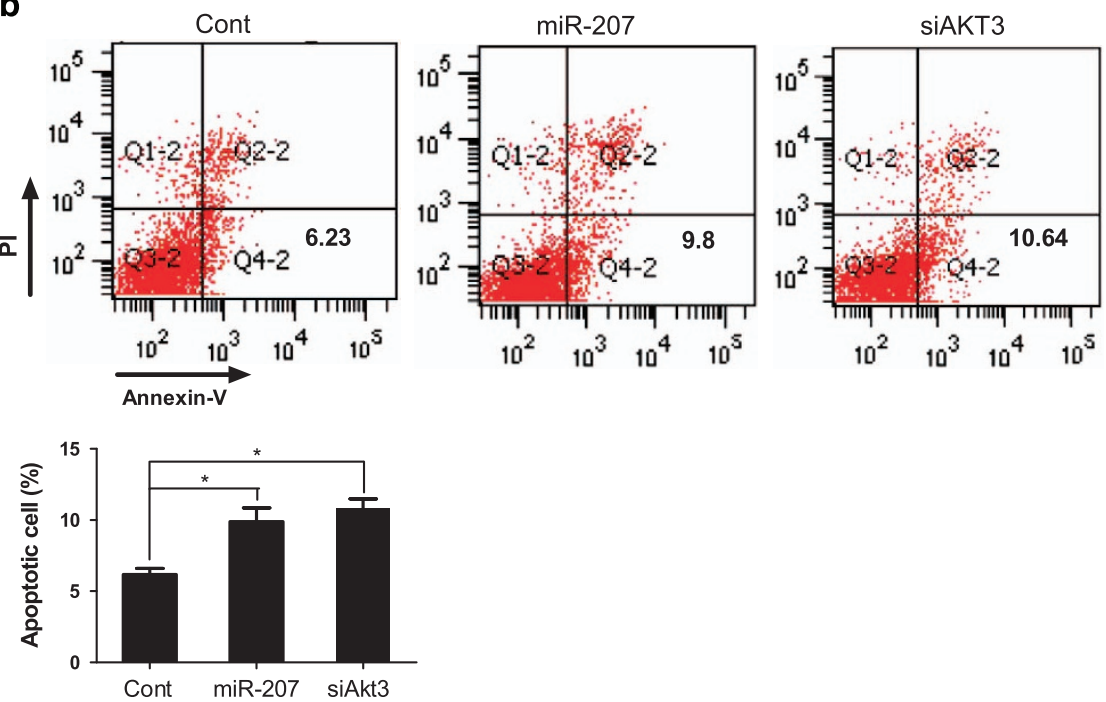

Figure 5 Downregulation of Akt3 mimics the effects of miR-207. (a) Expression levels of Akt3 and other Akt isoforms, such as Akt1 and Akt2, were determined using western blotting analyses in HEl-OC1 cells transfected with siAkt3 or control. (b) HEI-OC1 cells were transfected with miR-207, siAKT3 or control. The apoptosis assay using flow cytometry was performed $24 \mathrm{~h}$ after transfected cells were treated with 20 Gy IR. Error bars, S.D.; ${ }^{*} P \leq 0.001(n=3)$ versus control. (c) Expression of $\gamma$-H2AX, cleaved PARP and Akt3 proteins were determined by western blotting analyses using the indicated antibodies in transfected cells after $20 \mathrm{~Gy} I \mathrm{R}$. The cells used to determine the expression level of $\gamma$-H2AX and cleaved PARP and Akt3 were collected at $12 \mathrm{~h}$ and $24 \mathrm{~h}$ after IR, respectively

\section{Conclusions}

In summary, our study is the first to indicate that overexpression of miR-207 enhances apoptosis in irradiated auditory cells. Moreover, the enhancement of apoptosis is potentially caused by the disruption in DSBs repair. We also determined that specific inhibition of miR-207 significantly mitigated damages induced by IR on $\mathrm{HEI}-\mathrm{OC} 1$ cells, which may represent a novel strategy in protecting IR-induced SNHL. Furthermore, we confirmed Akt3 is a direct target for miR-207. Thus, additional studies are needed to clearly reveal the molecular mechanisms underlying the role of Akt3 in miR-207-enhanced apoptosis after IR.

\section{Materials and Methods}

Cell culture. The HEl-OC1 cell line was generously provided by F. Kalinec (House Ear Institute, Los Angeles, CA, USA). It is a conditionally immortalized organ of the Corti-derived epithelial cell line, ${ }^{30}$ which has been used to investigate the cellular and molecular mechanisms of ototoxicity induced by drugs, noise or irradiation. This cell line was maintained in high-glucose Dulbecco's modified Eagle's medium (Gibco, Cergy-Pontoise, France) containing $10 \%$ fetal bovine serum (Gibco) without antibiotics at $33{ }^{\circ} \mathrm{C}$ under $10 \% \mathrm{CO}_{2}$ in an incubator.

Animals. C57BL/6 mice were purchased from the Southern Medical University Laboratory. Animal care and killing were conducted according to methods approved by the Southern Medical University Animal Care and Use Committee, following guidelines of National Institute of Health for use of laboratory animals. $10 \mathrm{C57BL} /$ 6 mice were divided into two groups (control group and irradiation group).

Irradiation. The HEI-OC1 cells were irradiated at a distance of $100 \mathrm{~cm}$ from the source to the axis using a 6-MV linear accelerator (LINAC; 2300EX; Varian Co., Palo Alto, CA, USA) at a dose rate of $5.0 \mathrm{~Gy} / \mathrm{min}$. The mice of radiation group were placed and fixed in the prone position on a plate after they were anaesthetized with $40 \mathrm{mg} / \mathrm{kg} 0.1 \%$ pentobarbital sodium. The mice then received irradiation restricted to the head. A single dose of 20 Gy was delivered by opposed beams bilaterally with a distance of $100 \mathrm{~cm}$ from the source to the axis.

miRNA microarray. The expression of miRNAs in IR-treated versus untreated cells was analyzed using miRNA microarray. The total RNA was extracted from the untreated cells or cells $24 \mathrm{~h}$ post IR (20 Gy) using Trizol (Invitrogen, Carlsbad, CA, USA) according to the instructions provided by the manufacturer. Determination of the quantity and quality of the extracted RNA and the microarray experiment were performed by Guangzhou RiboBio Co. Ltd. using Mouse \& Rat miRNA OneArray V3.0 (Phalanx Biotech Company, Hsinchu, Taiwan).

qRT-PCR. After isolation from the IR-treated or untreated cells or cochlea, the total RNA was reverse-transcribed into cDNA using the PrimeScript RT reagent kit (TaKaRa, Dalian, China) according to the manufacturer's instructions. qPCR was performed using the ABI PRISM 7500 Fast Real-Time PCR System (Perkin Elmer/ Applied Biosystems, Rotkreuz, Switzerland) with a SYBR Premix Ex Taq II kit (TaKaRa). The small noncoding RNA U6 and housekeeping gene GADPH were 
used as an internal control for miRNA and Akt3 quantification, respectively. The sequences of the gene-specific primers used for qPCR are shown as follows: miR-207: forward 5'-ACACTCCAGCTGGGGCTTCTCCTGGCTCTCC-3'; miR-29c: forward 5'-ACACTCCAGCTGGGTAGCACCATTTGAAAT-3'; miR-466i-5p: forward 5'-ACACTCCAGCTGGGTGTGTGTGTGTGTG-3'; universal reverse primer for miRNAs: 5'-TGGTGTCGTGGAGTCG-3'; Akt3: forward 5'-ACCGCACACGTTTC TATGGT-3', reverse 5'-CCCTCCACCAAGGCGTTTAT-3'; U6: forward 5'-CTC GCTTCGGCAGCACA-3', reverse 5'-AACGCTTCACGAATTTGCGT-3'; GADPH: forward 5'-GGATTTGGTCGTATTGGG-3', reverse 5'-GTGGCTGGGGCTCTACTTC-3', All reactions were performed in triplicate for each sample.

Northern blotting. The locked nucleic acid (LNA) probe for miR-207 labeled with digoxigenin (DIG Oligonucleotide 3'-End Labeling Kit; Roche, Stockholm, Sweden) were purchased from Exiqon Co (Vedbaek, Denmark). U6 RNA was detected using a DIG-labeled U6 DNA probe. Northern blotting was performed as described. ${ }^{31}$ Total RNA from cochleas was resolved by $15 \%$ denaturing polyacrylamide gel electrophoresis and transferred by electroblotting to membranes. Blots were pre-hybridized and hybridized by overnight incubation in buffer containing $0.2 \mathrm{nM}$ DIG-labeled probes at $52{ }^{\circ} \mathrm{C}$ for miR-207 LNA probe or $25^{\circ} \mathrm{C}$ for the U6 DNA probe. Blots were stringently washed twice in SDS for 30 min at $52^{\circ} \mathrm{C}$ for miR-207 LNA probe or $25^{\circ} \mathrm{C}$ for the U6 DNA probe, rinsed in wash buffer, and incubated in block buffer for $30 \mathrm{~min}$. Subsequently, blots were incubated with antiDIG-AP Fab fragment in block buffer for $1 \mathrm{~h}$, washed in wash buffer and detection buffer separately. Anti-DIG-AP was detected using CDP-star chemiluminescent substrate for alkaline phosphatase (AP). Blots were stripped by incubation at $70^{\circ} \mathrm{C}$ in $0.1 \times$ SSC containing $1 \%$ SDS and probed up to three times.

Parafin section ISH. ISH was performed to confirm the expression of miR-207 in cochlea hair cells in vivo. Cochleas of C57BL/6 mice were carefully and quickly resected, followed by removing the stapes from the oval window and opening the cochlea apex. We then placed the cochleas in fresh $4 \%$ paraformaldehyde (PFA) in PBS overnight at $4{ }^{\circ} \mathrm{C}$. After decalcification with $0.1 \mathrm{M}$ ethylenediaminetetraacetic for 3 days at $4^{\circ} \mathrm{C}$, the cochleas were embedded in paraffin and sectioned at $5 \mu \mathrm{m}$. ISH was performed as described. ${ }^{32}$ Briefly, sections were hybridized with labeled LNA probe, which was detected using AP-conjugated sheep anti-DIG Fab fragment and BM Purple AP Substrate (Roche). The sections were then fixed by 4\% PFA for 20 min finish with milliQ water (Millipore, Billerica, MA, USA) rinses. Slides were coverslipped with Aquamount (Merck, Darmstadt, Germany). The slides were observed using a light microscope.

Transfection. MiR-207 mimics, miR-207 inhibitor, miR-29c mimics, miR-466i-5p mimics, siAkt3 and control were purchased from RiboBio Co. After the cells were seeded in six-well plates, transfection was performed using Lipofectamine 2000 (Invitrogen) according to the instructions provided by the manufacturer. Experiments performed on transfected cells were carried out $48 \mathrm{~h}$ post transfection.

MTT assay. This assay was performed to examine the viability of $\mathrm{HEI}-\mathrm{OC} 1$ cells. The transfected cells were seeded in 96-well plates at a density of $1 \times 10^{4}$ cells/well followed by radiation treatment. Next, $20 \mu \mathrm{l}$ of $5 \mathrm{mg} / \mathrm{ml}$ MTT (Sigma, St. Louis, MO, USA) was added to each well at $48 \mathrm{~h}$ post IR (10,20 Gy) or without IR treatment. After culturing for $4 \mathrm{~h}$, the medium was replaced with $150 \mu$ l/well dimethylsulfoxide (Sigma) and vortexed for $10 \mathrm{~min}$. The optical density value was measured at $490 \mathrm{~nm}$ and the wells that did not contain cells that were used as blanks. Each experiment was performed in triplicate.

Immunofluorescent staining for $\gamma$-H2AX. We estimated the DNA damage by performing immunofluorescent staining for $\gamma$-H2AX. MiR-207-mimicstransfected, miR-207-inhibitor-transfected and control-transfected cells were grown on polylysine-coated coverglasses. At $12 \mathrm{~h}$ after radiation ( $20 \mathrm{~Gy})$, the cells were fixed in $4 \%$ PFA for $30 \mathrm{~min}$, permeabilized in $0.25 \%$ triton $\mathrm{X}-100$ and blocked for $30 \mathrm{~min}$ in $1 \%$ goat serum. The cells were then incubated overnight at $4{ }^{\circ} \mathrm{C}$ with anti$\gamma$ H2AX primary antibody (1:100 dilution; Abcam, San Francisco, CA, USA). Next, the cells were washed in PBS, and rabbit anti-mouse AlexaFlour-488 secondary antibody (1:200 dilution; Abcam) was applied for $1 \mathrm{~h}$ at room temperature in the dark. After three 5-min washes in PBS, the samples were mounted in fluorescence mounting medium with DAPI. The cells were observed using a fluorescence microscope (Olympus, Shinjuku-ku, Tokyo, Japan), and the $\gamma$-H2AX foci were manually quantified in at least three individual fields of $\sim 100$ cells.
Cell cycle analysis using flow cytometry. The transfected cells were collected at $24 \mathrm{~h}$ after IR (20 Gy) or without IR. Next, cells were washed with PBS once and resuspended in PBS containing $0.2 \%$ Triton X-100 for permeabilization and propidium iodide (PI) staining. After incubation for $30 \mathrm{~min}$, the cells were analyzed using flow cytometry. Each experiment was performed in triplicate.

Apoptosis analysis using flow cytometry. The transfected cells were collected at $24 \mathrm{~h}$ post IR (20 Gy) or without IR treatment. Cells undergoing apoptosis were determined by staining with Alexa Fluor 488 annexin $\mathrm{V}$ and PI according to the protocol provided by the manufacturer (Invitrogen). Each experiment was performed in triplicate.

Western blotting. Cells were collected and western blot analyses were performed as previously described. ${ }^{33} \beta$-actin was used as the loading control. The primary antibodies used for western blotting included: rabbit anti-cleaved PARP (\#9544, Cell Signaling, Beverly, MA, USA), anti-H2AX (\#9718, Cell Signaling), antiAkt1 (\#9514, SBA), anti-Akt2 (\#8715, SBA), anti-Akt3 (\#4059, Cell Signaling), antipAkt (\#4060, Cell Signaling) and anti- $\beta$-actin (\#4970, Cell Signaling).

Bioinformatic analyses. We obtained the mature sequence of miR-207 from the miRNA database (http://www.mirbase.org/). EIMMo (http://www.mirz.unibas.ch/ EIMMo2/), miRanda (http://www.microrna.org), miTarget (http://cbit.snu.ac.kr/ $\sim$ miTarget/), PicTar (http://pictar.mdc-berlin.de/) and TargetScan (http://www. targetscan.org/) was used to predict the target gene of miR-207.

Reporter plasmid constructs and luciferase reporter assays. We confirmed the direct target of miR-207 by performing this assay. The $3^{\prime}$-UTR of the Akt3 gene containing the predicted target sites of miR-207 was amplified by PCR using the primers $5^{\prime}$-TCACAGATCATTGCCTGCGT-3' for Akt3-3'-UTRforward and 5'-TCCCACACCTCGGTTCTACT-3' for Akt3-UTR-reverse was cloned into the Renilla luciferase gene (pLUC-REPORT vector, Promega, Madison, WI, USA). A mutant 3'-UTR of Akt3 with a mutated sequence (5'-...AAUCUGUGCCUCUUCAGAUGCUGUGA...-3', the mutated sites are underlined) in the complementary site for the miR-207 seed region was also amplified and cloned into the pLUC-REPORT vector. For the reporter luciferase assay, HEI-OC1 calls were cultured in 96-well plates and co-transfected with pLUC-3'UTR-Akt3 (Akt3-WT) or pLUC-3'UTR-Mut-Akt3 (Akt3-Mut) and miR-207 mimics or control with Lipofectamine 2000 (Life Technologies, Carlsbad, CA, USA). Forty-eight hours after transfection, cells were assayed for luciferase activity with the Dual-Luciferase Assay System (Promega) according to the manufacturer's instructions. Independent assays were performed in triplicate.

Statistical analyses. Data were analyzed using the Student's $t$-test or oneway ANOVA for statistical significance. Statistical evaluations are presented as the mean \pm S.D., and a $P$-value of $<0.05$ was considered to be statistically significant.

\section{Conflict of Interest}

The authors declare no conflict of interest.

Acknowledgements. We acknowledge the excellent technical support and sincere help of the Functional Genomics and Bioinformatics cores of Southern Medical University. We also thank Prof. F Kalinec (House Ear Institute, Los Angeles, CA, USA) for kindly providing the HEI-OC1 cells. This study was supported by the National Natural Science Foundation of China (81272508, 81302385), Specialized Research Fund for the Doctoral Program of Higher Education of China (20114433110015), Guangzhou Science and Technology Project Fund (11C22120714) and Guangdong Provincial Department of education research project (2013KJCX0038).

1. Tan PX, Du SS, Ren C, Yao QW, Yuan YW. Radiation-induced cochlea hair cell death: mechanisms and protection. Asian Pac J Cancer Prev 2013; 14: 5631-5635.

2. Li JJ, Guo YK, Tang QL, Li SS, Zhang XL, Wu PA et al. Prospective study of sensorineural hearing loss following radiotherapy for nasopharyngeal carcinoma. J Laryngol Otol 2010; 124: 32-36.

3. Low WK, Burgess R, Fong KW, Wang DY. Effect of radiotherapy on retro-cochlear auditory pathways. Laryngoscope 2005; 115: 1823-1826. 
4. Bohne BA, Marks JE, Glasgow GP. Delayed effects of ionizing radiation on the ear. Laryngoscope 1985; 95: 818-828.

5. Linskey ME, Johnstone PA. Radiation tolerance of normal temporal bone structures: implications for gamma knife stereotactic radiosurgery. Int J Radiat Oncol Biol Phys 2003; 57: 196-200.

6. Honore HB, Bentzen SM, Moller K, Grau C. Sensori-neural hearing loss after radiotherapy for nasopharyngeal carcinoma: individualized risk estimation. Radiother Oncol 2002; 65: 9-16.

7. Low WK, Tan MG, Sun L, Chua AW, Goh LK, Wang DY. Dose-dependant radiation-induced apoptosis in a cochlear cell-line. Apoptosis 2006; 11: 2127-2136.

8. Fleury B, Lapeyre M. [Tolerance of normal tissues to radiation therapy: ear]. Cancer Radiother 2010; 14: 284-289.

9. Sha SH, Taylor R, Forge A, Schacht J. Differential vulnerability of basal and apical hair cells is based on intrinsic susceptibility to free radicals. Hear Res 2001; 155: 1-8.

10. Pyun JH, Kang SU, Hwang HS, Oh YT, Kang SH, Lim YA et al. Epicatechin inhibits radiationinduced auditory cell death by suppression of reactive oxygen species generation. Neuroscience 2011; 199: 410-420.

11. Low WK, Sun L, Tan MG, Chua AW, Wang DY. L-N-Acetylcysteine protects against radiationinduced apoptosis in a cochlear cell line. Acta Otolaryngol 2008; 128: 440-445.

12. Mujica-Mota MA, Salehi P, Devic S, Daniel SJ. Safety and otoprotection of metformin in radiation-induced sensorineural hearing loss in the guinea pig. Otolaryngol Head Neck Surg 2014; 150: 859-865.

13. Jackson RJ, Standart N. How do microRNAs regulate gene expression?. Sci STKE 2007; 2007: re1.

14. Ambros V. The functions of animal microRNAs. Nature 2004; 431 : 350-355.

15. Lhakhang TW, Chaudhry MA. Current approaches to micro-RNA analysis and target gene prediction. J Appl Genet 2012; 53: 149-158.

16. Weston MD, Pierce ML, Jensen-Smith HC, Fritzsch B, Rocha-Sanchez S, Beisel KW et al. MicroRNA-183 family expression in hair cell development and requirement of microRNAs for hair cell maintenance and survival. Dev Dyn 2011; 240: 808-819.

17. Ushakov K, Rudnicki A, Avraham KB. MicroRNAs in sensorineural diseases of the ear. Front Mol Neurosci 2013; 6: 52.

18. Wang Z, Liu Y, Han N, Chen X, Yu W, Zhang W et al. Profiles of oxidative stress-related microRNA and mRNA expression in auditory cells. Brain Res 2010; 1346: 14-25.

19. Patel M, Cai Q, Ding D, Salvi R, Hu Z, Hu BH. The miR-183/Taok1 target pair is implicated in cochlear responses to acoustic trauma. PLOS One 2013; 8: e58471.

20. Yu L, Tang H, Jiang XH, Tsang LL, Chung YW, Chan HC. Involvement of calpain-I and microRNA34 in kanamycin-induced apoptosis of inner ear cells. Cell Biol Int 2010; 34: 1219-1225.

21. Chaveles I, Zaravinos A, Habeos IG, Karavias DD, Maroulis I, Spandidos DA et al. MicroRNA profiling in murine liver after partial hepatectomy. Int J Mol Med 2012; 29: 747-755.

22. Li L, Chen HZ, Chen FF, Li F, Wang M, Wang L et al. Global microRNA expression profiling reveals differential expression of target genes in 6-hydroxydopamine-injured MN9D cells. Neuromolecular Med 2013; 15: 593-604.
23. Selzer E, Hebar A. Basic principles of molecular effects of irradiation. Wien Med Wochenschr 2012; 162: 47-54.

24. Franken NA, ten Cate R, Krawczyk PM, Stap J, Haveman J, Aten J et al. Comparison of RBE values of high-LET alpha-particles for the induction of DNA-DSBs, chromosome aberrations and cell reproductive death. Radiat Oncol 2011; 6: 64.

25. Stahl JM, Sharma A, Cheung M, Zimmerman M, Cheng JQ, Bosenberg MW et al. Deregulated Akt3 activity promotes development of malignant melanoma. Cancer Res 2004; 64: 7002-7010.

26. Nassirpour R, Mehta PP, Yin MJ. miR-122 regulates tumorigenesis in hepatocellular carcinoma by targeting AKT3. PLOS One 2013; 8: e79655.

27. Bozulic L, Surucu B, Hynx D, Hemmings BA. PKBalpha/Akt1 acts downstream of DNA-PK in the DNA double-strand break response and promotes survival. Mol Cell 2008; 30: 203-213.

28. Toulany M, Kehlbach R, Florczak U, Sak A, Wang S, Chen J et al. Targeting of AKT1 enhances radiation toxicity of human tumor cells by inhibiting DNA-PKcs-dependent DNA double-strand break repair. Mol Cancer Ther 2008; 7: 1772-1781.

29. Sahlberg SH, Gustafsson AS, Pendekanti PN, Glimelius B, Stenerlow B. The influence of AKT isoforms on radiation sensitivity and DNA repair in colon cancer cell lines. Tumour Biol 2013; 35: 3525-3534.

30. Kalinec GM, Webster P, Lim DJ, Kalinec F. A cochlear cell line as an in vitro system for drug ototoxicity screening. Audiol Neurootol 2003; 8: 177-189.

31. Weston MD, Pierce ML, Rocha-Sanchez S, Beisel KW, Soukup GA. MicroRNA gene expression in the mouse inner ear. Brain Res 2006; 1111: 95-104.

32. Kloosterman WP, Wienholds E, de Bruijn E, Kauppinen S, Plasterk RH. In situ detection of miRNAs in animal embryos using LNA-modified oligonucleotide probes. Nat Methods 2006; 3: 27-29.

33. Du $S$, Yao $Q$, Tan $P$, Xie G, Ren $C$, Sun $Q$ et al. Protective effect of tanshinone IIA against radiation-induced ototoxicity in $\mathrm{HEI}-\mathrm{OC} 1$ cells. Oncol Lett 2013; 6: 901-906.

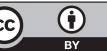

Cell Death and Disease is an open-access journal published by Nature Publishing Group. This work is licensed under a Creative Commons Attribution 4.0 International Licence. The images or other third party material in this article are included in the article's Creative Commons licence, unless indicated otherwise in the credit line; if the material is not included under the Creative Commons licence, users will need to obtain permission from the licence holder to reproduce the material. To view a copy of this licence, visit http://creativecommons.org/licenses/by/4.0 